\title{
Performance Analysis of Antenna Array Calibration and its Impact on Beamforming: A Survey
}

\author{
Chen Zhang, Jiqing Ni, Yantao Han \\ Research Institute of Communication Technology \\ Beijing Institute of Technology University (BIT) \\ Beijing, China \\ E-mail: \{20905250,njq, autumnwind\}@bit.edu.cn
}

\author{
GaoKe Du \\ Huawei Company \\ Beijing, China \\ E-mail: \{Gaoke.Du\}@huawei.cn
}

\begin{abstract}
Multiple antennas techniques have been widely adopted in broadband wireless communication systems to enhance spectral efficiency and achieve space diversity gain. Transmitter beamforming is one of promising multiple antenna techniques for IMT-Advanced system, which takes advantage of directional signal transmission or reception. For efficient transmitter beamforming, TDD (Time Division Duplex) system has an inherit advantage to exploit the channel reciprocity between uplink and downlink, and no additional channel feedback is needed. However, the mismatches of actual RF (ratio frequency) channel may cause channel non-reciprocity. This paper presents the latest progress on multiple antenna calibration, including the analysis of the hardware error characteristics, typical statistical models of the calibration error (CE), and the impacts on downlink beamforming due to CE. Furthermore, we present the performance analysis and comparison for widely used Base Station (BS) based calibration schemes. Finally, several possible terminal based calibration schemes are summarized to compensate the actual RF mismatches.
\end{abstract}

Keywords-Antenna array calibration; Gain and phase mismatches; Calibration error; channel reciprocity; TDD

\section{INTRODUCTION}

In MIMO system, the availability of channel state information (CSI) at transmitter can help the transmitter to shape the transmitted signal to improve the overall system spectral efficiency. There are two approaches to obtain CSI at transmitter [1]. The first approach is that the receiver estimates CSI and sends it back to the transmitter through the feedback channel. In order to obtain accurate CSI at transmitter, substantial channel estimations and feedbacks are needed, which result in high overhead and low spectral efficiency. The other approach is based on channel reciprocity principle in TDD system. The reciprocity principle refers to that uplink and downlink have the same channel response because they are operated on the same frequency band in TDD system. As a result, the transmitter can utilize the reverse CSI instead of the forward CSI to perform appropriate transmit beamforming.

However, the RF paths will result in the non-reciprocity of the measured channel (effective channel). Effective channel usually consists of three parts [2]: the RF path at the transmitter, the propagation channel and the RF path at the receiver. If the time interval between uplink and downlink is less than the channel coherence time, the propagation channel is reciprocal. However, different RF chains are used in reception and transmission for each antenna. So there is no reason to assume that the RF path is reciprocal. Accordingly, the effective channel is non-reciprocal. Hence, in order to fully exploit the channel reciprocity in TDD system to obtain more accurate CSI and improve system throughput, calibrations at UE and at $\mathrm{BS}$ are indispensable.

So far, a lot of works have been done in hardware calibration, including physical characteristics of hardware error [3], the calibration method and performance loss due to CE. In the literature, many papers have pointed out that hardware error is mainly derived from imperfect analog devices, and it depends on surrounding environments [4]. And other papers have considered different methods to benefit from channel reciprocity in TDD system. One method is absolute calibration which requires an external reference source with tight requirements [5] or a specially crafted transceiver [6]. Another method is relative calibration which relies on a calibration phase to estimate the difference between uplink (UL) and downlink (DL) [2]. Compared to the former, the relative calibration method is more attractive due to its low cost and no feedback channel. Now, the calibration at BS is used in TDSCDMA network. Because the CE on mobile terminal will lead to performance degradation, the researches on UE calibration have carried out by many companies [7]-[9]. Based on extensive used calibration error statistical models, this paper analyzes the CEs' impact by simulation and sums up several feasible mitigation methods for UE.

This paper is organized as follows. In section II, the system model and the calibration error statistical models are presented. And then in section III, impacts on DL BF from calibration errors are analyzed and compared. In section IV, several possible terminal based calibration methods are summarized, and finally the conclusion is given in section $\mathrm{V}$.

\section{System MOdEl AND CALIBRATION ERROR STATISTICAL MODELS}

Considering a TDD MIMO system, in which the BS has M transmit elements, and the UE has $\mathrm{N}$ receive elements. Fig 1 
shows the transmission model between the BS's reference antenna $i$ and the UE's reference antenna $j$.

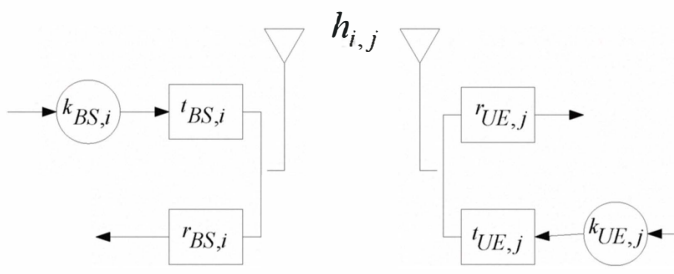

Figure 1. Transmission model between antenna $i$ of BS and antenna $j$ of UE in TDD system

In Fig.1, the propagation channel between the two antennas is reciprocal and it can be described by its frequency response $h_{i, j} \cdot t_{B S, i}$ and $r_{B S, i}$ are RF factors respectively in Tx chain and Rx chain for antenna $i(i=1 \ldots \mathrm{M})$ at BS. Similarly, $t_{U E, j}$ and $r_{U E, j}$ are respectively RF factor in $\mathrm{Tx}$ chain and $\mathrm{Rx}$ chain for the $j$-th $(j=1 \ldots \mathrm{N})$ antenna at UE. $k_{B S, i}$ and $k_{U E, j}$ are complex factors representing the calibration factor at $\mathrm{BS}$ and $\mathrm{UE}$, respectively. In order to use channel reciprocity in $M \times N$ MIMO system, we need to have [10]

$$
\begin{aligned}
& \frac{k_{B S, 1} t_{B S, 1}}{r_{B S, 1}}=\frac{k_{B S, 2} t_{B S, 2}}{r_{B S, 2}}=\cdots=\frac{k_{B S, M} t_{B S, M}}{r_{B S, M}} \\
& \frac{k_{U E, 1} t_{U E, 1}}{r_{U E, 1}}=\frac{k_{U E, 2} t_{U E, 2}}{r_{U E, 2}}=\cdots=\frac{k_{U E, N} t_{U E, N}}{r_{U E, N}}
\end{aligned}
$$

However, if the BS and the terminal don't meet (1) and (2), there are calibration errors $C_{B S}$ and $C_{U E}$ at BS and at terminal respectively. $C_{B S}$ and $C_{U E}$ are diagonal matrix with diagonal complex elements and consist of amplitude error and phase error[11]. Furthermore, matrix $C_{B S}$ and $C_{U E}$ can be expressed as below

$$
\begin{aligned}
& C_{B S}=\operatorname{diag}\left[c_{B S, 1}, \cdots c_{B S, M}\right]=\operatorname{diag}\left[\alpha_{B S, 1}, \cdots \alpha_{B S, M}\right] \operatorname{diag}\left|e^{j \beta_{B S, M}}, \cdots e^{j \beta_{B S, M}}\right|(3) \\
& C_{U E}=\operatorname{diag}\left[c_{U E, 1}, \cdots c_{U E, N}\right]=\operatorname{diag}\left[\alpha_{U E, 1} \cdots \alpha_{U E, N}\right] \operatorname{diag}\left[e^{j \beta_{U E, 1}}, \cdots e^{j \beta_{U E, N}}\right](4)
\end{aligned}
$$

Where $\alpha$ and $\beta$ represent amplitude error and phase error respectively and

$$
\begin{gathered}
c_{B S, i}=\frac{k_{B S, i} t_{B S, i}}{r_{B S, i}}, \quad i=1,2 \cdots M \\
c_{U E, i}=\frac{k_{U E, i} t_{U E, i}}{r_{U E, i}}, \quad i=1,2 \cdots N
\end{gathered}
$$

Generally, the elements of calibration error matrix are assumed to be i.i.d random variables. There are two methods to model the antenna CE. Taking the errors at BS as an example, one approach is modeling $c_{B S, 1}, \cdots c_{B S, M}$ directly, and the other approach is modeling $\alpha_{B S, 1}, \cdots \alpha_{B S, M}$ and $\beta_{B S, 1}, \cdots \beta_{B S, M}$ respectively. Three common used error statistical models are given by TABLE I. [7][9]. Where $\mathrm{N}$ means complex normal distribution in model 1 and means real normal distribution in model 2 and model 3. U means uniform distribution. $\delta$ and $\varphi$ indicate the level of CE.

TABLE I. ThreE KindS OF ERror StATISTICAL MODELS

\begin{tabular}{|c|c|}
\hline $\begin{array}{c}\text { Error Statistical } \\
\text { Model }\end{array}$ & \multicolumn{1}{|c|}{ Distribution } \\
\hline Model 1 & $c_{B S, i} \sim N\left(1, \delta_{B S}^{2}\right), \quad i=1,2 \cdots M$ \\
\hline Model 2 & $\alpha_{B S, i} \sim N\left(1, \delta_{B S, \alpha}^{2}\right), \quad i=1,2 \cdots M ;$ \\
& $\beta_{B S, j} \sim N\left(0, \delta_{B S, \beta}^{2}\right), j=1,2 \cdots N$ \\
\hline Model 3 & $10 \lg \alpha_{B S, i} \sim N\left(0, \delta_{B S, \alpha}^{2}\right), i=1,2 \cdots M$ \\
& $\beta_{B S, j} \sim U\left[\begin{array}{ll}-\varphi & \varphi\end{array}\right], j=1,2 \cdots N, \varphi \in(0, \pi]$ \\
\hline
\end{tabular}

\section{PERformance ANALysis With CALIBration ERrors}

After the implementation of the calibration, the effective uplink and downlink channel responses are $H_{U, C}$ and $H_{D, C}$ respectively. According to the principle of the SVD-based downlink open-loop beamforming, the transpose of effective UL MIMO channel matrix can be further expressed as below.

$$
H_{U, C}^{T}=U \Sigma V^{H}
$$

Where $(\bullet)^{H}$ denotes the conjugate and transpose operation. And the matrices $\mathrm{U}, \mathrm{V}$ are unitary of dimensions $N \times N$ and $M \times M$, while $\Sigma$ is non-negative and diagonal with diagonal elements $\sqrt{\lambda_{i}}, i=1,2 \cdots l$, which are the singular values of matrix $H_{U, C}^{T}$ and $l$ is the rank of $H_{U, C}^{T}$. Then, the precoding vectors are the first $L$ columns of matrix $\mathrm{V}$, which are the eigenvectors corresponding to the $\mathrm{L}$ maximum singular values of $H_{U, C}^{T} . L$ is the number of the data streams.

When there are calibration error $C_{B S}$ and $C_{U E}, H_{D, C}$ and $H_{U, C}$ will meet the follow equation

$$
H_{D, C}=C_{U E} H_{U, C}^{T} C_{B S}
$$

By using (3), (4) and (7), the equation (8) can be written as (9). 


$$
H_{D, C}=\left[\begin{array}{lll}
\alpha_{U E, 1} & & \\
& \ddots & \\
& & \alpha_{U E, n}
\end{array}\right]\left[\begin{array}{ccc}
e^{j \beta_{U E, 1}} & & \\
& \ddots & \\
& & e^{j \beta_{U E, n}}
\end{array}\right] U \Sigma V^{H}\left[\begin{array}{lll}
\alpha_{B S, 1} & & \\
& \ddots & \\
& & \alpha_{B S, m}
\end{array}\right]\left[\begin{array}{lll}
j \beta_{B S, 1} & & \\
& \ddots & \\
& & e^{j \beta_{B S, m}}
\end{array}\right]
$$

From (9), amplitude error at UE and BS will lead to different channel properties [9] between $H_{D, C}$ and $H_{U, C}$, i.e. different eigen-values, which will impact on the decision of the rank number for each channel, and the decision of MCS levels for data transmission. In addition, with the phase error at UE, eigen-values and right side eigenvectors of $H_{D, C}$ and $H_{U, C}$ are completely same, while the phase error at BS will result in different right side eigenvectors. So the phase error at BS will degenerate beamforming performance, but the phase error at UE don't.

Many literatures and 3GPP proposals have investigated the performance impact from $\mathrm{Tx} / \mathrm{Rx}$ mismatch at $\mathrm{BS}$ and UE. As far as CE at BS is concerned, it is close to unity amplitude wise so the phase error will be dominant. And the CE at BS has much more impacts on performance than that at UE. So antenna array calibration is required at BS in LTE system, while the calibration at UE is still in evaluation stage. So it is necessary to further assess the impacts on system performance from terminal CE. Here, assuming calibration between $\mathrm{Tx}$ chain and Rx chain for each antenna at BS was perfectly done, throughput comparisons with different assumption of $\mathrm{CE}$ are evaluated in DL dual layer BF transmission [9]. TABLE II. lists simulation parameters.

TABLE II. SIMULATION PARAMETERS

\begin{tabular}{|c|c|}
\hline Parameters & Assumption \\
\hline Carrier Frequency & $2.0 \mathrm{GHz}$ \\
\hline System Bandwidth & $5 \mathrm{MHz}$ \\
\hline UE Speed & $3 \mathrm{~km} / \mathrm{h}$ \\
\hline Number of Antennae & $8 \times 2$ \\
\hline Channel Model & SCM-C (dual polarization) \\
\hline SRS Estimation Loss & Ideal \\
\hline CodeWord & 2 \\
\hline Number of Layers & 2 \\
\hline AMC & $\begin{array}{l}\text { CQI calculation based on UL channel in } \\
\text { TDD and DL noise level. }\end{array}$ \\
\hline CQI Delay (ms) & 1 \\
\hline Rank Adaption & fixed rank $=2$ \\
\hline Harq & $\mathrm{NO}$ \\
\hline Number of PRBs & 4 (scheduling granularity) \\
\hline $\begin{array}{c}\text { Granularity of } \\
\text { Beamforming (RB) }\end{array}$ & 1 \\
\hline $\begin{array}{c}\text { Subband Bandwidth } \\
\text { of CQI(RB) }\end{array}$ & 4 \\
\hline Overhead & $\begin{array}{c}\text { Control region: } 3 \text { OFDM symbols per } 1 \mathrm{~ms} \\
\text { subframe } \\
\text { CRS: port } 0 \sim \text { port } 3 \text { as LTE R8 } \\
\text { DRS: } 12 \text { REs for a pair of PRB }\end{array}$ \\
\hline
\end{tabular}

In Fig. 2, amplitude calibration factor $\alpha_{U E, i}, i=1,2 \cdots N$ are assumed to be of log-normal distribution, i.e. $10 \log a_{U E, i} \sim N\left(0, \delta_{U E, \alpha}^{2}\right)$, and phase calibration factor $\beta_{U E, i}$,

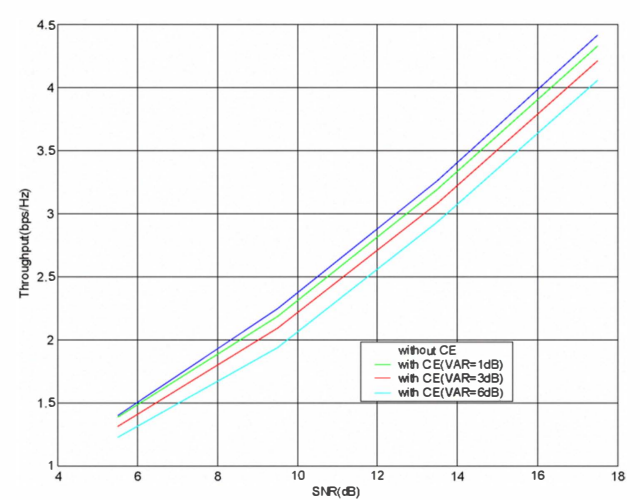

Figure 2. Throughput comparison with amplitude calibration errors in dual layer BF

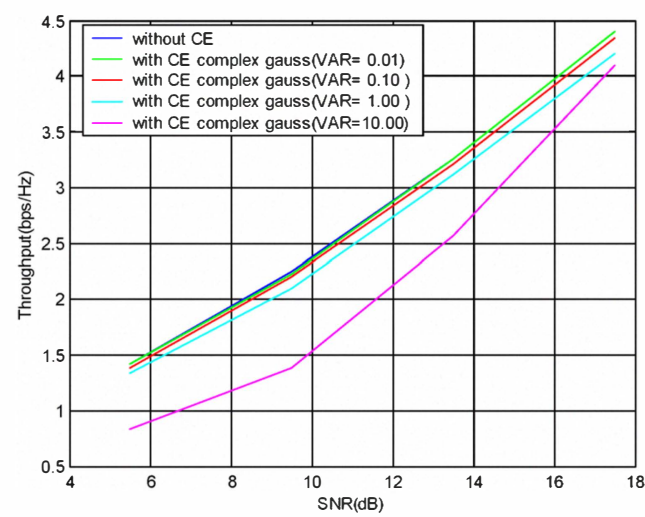

Figure 3. Throughput comparison with complex gauss $\mathrm{CE}$ in dual layer $\mathrm{BF}$

$i=1,2 \cdots N$, are assumed to be of uniform distribution within $\left[\begin{array}{ll}-\pi / 6 & \pi / 6\end{array}\right]$. And it shows that,

- With $\mathrm{CE}$ variance of $6 \mathrm{~dB}$, it will incur nearly $12 \%$ throughput loss compared with those without $\mathrm{CE}$ at median SNR of $6 \mathrm{~dB}$ and $10 \mathrm{~dB}$, and it will incur nearly $8 \%$ throughput loss at SNR of $16 \mathrm{~dB}$.

- With the reduction of CE variance, it will incur less performance loss.

It will incur nearly $0.2 \mathrm{~dB}$ and $0.6 \mathrm{~dB}$ SNR penalty loss respectively with $\mathrm{CE}$ variance of $1 \mathrm{~dB}$ and $3 \mathrm{~dB}$. With $\mathrm{CE}$ variance of $6 \mathrm{~dB}$, it will incur nearly $1.2 \mathrm{~dB}$ SNR penalty loss.

In Fig. 3, CEs are assumed to be i.i.d complex Gaussian random variables with mean value of 1 and variance of $\delta^{2}$. And it shows that, 
- The throughput curve with CE variance of 0.01 nearly approximates to that without $\mathrm{CE}$.

- With the increase of CE variance, it will incur much performance loss.

It will bring as much as nearly $0.7 \mathrm{~dB}$ SNR penalty loss with $C E$ variance of 1 . With $C E$ variance of 10 , as much as 4 $\mathrm{dB}$ SNR penalty losses can be observed within median SNR range, and it will incur less SNR penalty loss within high SNR range.

From all the above simulation, even with fixed rank 2, i.e. rank mismatch due to amplitude calibration error is not considered, it will still result in throughput loss due to MCS level mismatch caused by the same reason.

\section{CALIBRATION MeTHOdS}

There are two kinds of calibration methods. One is absolute calibration relying on hardware solutions. Another is relative calibration which uses conventional channel estimation and feedback to estimate the relationship between forward channel and reverse channel. The absolute calibration is not cost effective in the implementation and commercialization point of view since it requires external hardware with tight requirement. On the contrary, the relative calibration does not introduce additional hardware devices and is implemented entirely in signal space. With these properties it's suggested that the relative calibration be used into the commercial TDD system. In this section, we present the analyses and comparisons for the relative calibration.

The relative calibration establishes the relationship between the effective channel in both directions using traditional channel measurement and feedback technology during the calibration phase. Taking terminal calibration for example, the principle of the relative calibration is as follows [2]:

- The terminal to be calibrated selects a supporter (s), through which implements the calibration.

- The terminal transmits calibration signal to the supporter, and the supporter estimates the effective channel frequency response of the forward link $h_{f}$, and feeds it back to the terminal.

- The supporter transmits calibration signal to the terminal, and the terminal estimates the effective channel frequency response of the reverse link $h_{r}$.

- The terminal compares $h_{f}$ and $h_{r}$, and computes the calibration factor using $k=h_{r} / h_{f}$.

However, in wideband TDD system, due to selective fading environments, the calibration factor $k$ usually depends on frequency [10]. So, in this case, the above relative calibration should be operated in specific frequency. In addition, for OFDMA/TDD system, we can select some sub-bands or frequencies of good channel conditions, and then perform the relative calibration at the selected sub-bands. Finally, the calibration parameters are interpolated in frequency domain for whole bandwidth.

Many existing patents [15]-[16] and 3GPP proposals have proposed different implementation methods based on relative calibration, which can be divided into two categories according to the kind of calibration signals. One is based on dedicated test signal. This method is simple, flexible and has high calibration accuracy. The other one uses transmitting signals to obtain calibration coefficients [13], which requires a special transceiver and is not flexible. Most of the methods given in the literature use the dedicated test signals. As the calibration at $\mathrm{BS}$ is required in TDD system, based on the existing literature, we summarize several possible terminal based calibration schemes to compensate the actual RF mismatches.

For calibration schemes using dedicated test signals, the terminal can compensate the RF mismatches either through adding a calibration circuit in the terminal or using a calibration transmitter/receiver in addition to the apparatus.

\section{A. Self-calibration}

Self-calibration compensates the RF mismatches within the terminal [16], and does not require external equipment. But adding relevant circuitry in the UE may be necessary, which is an attenuator connecting the antenna of the terminal.

The procedure of the self-calibration is as follows. Firstly, the terminal selects an antenna as a reference antenna, whose calibration factor is set to 1 . And then the other antennas calibrate with the reference antenna, through the time division multiplexing, using the relative calibration algorithm. In this case, the calibration accuracy depends on the reciprocity of the attenuator. For more than two antennas calibration, there is an improved method [12]. Taking three antennas calibration for example, its procedure is as follows:

- Selecting antenna 1 as reference antenna, the calibration coefficients obtained using self-calibration are $c 1$ _ant $1, c 2_{\text {_ant } 1,}, 3_{\text {_ant } 1}$.

- Selecting antenna 2 as reference antenna, the calibration coefficients obtained using self-calibration are $c 1$ ant $2, c 2$ _ant $2, c 3$ _ant 2 .

$$
\begin{aligned}
& \text { The final calibration } \\
& c 1=c 1 \text { coefficients ant } 2 \times \frac{c 3 \text { ant } 1}{c 3 \text { ant } 2}, \quad c 2=c 2_{\text {ant }} \text { ant } \\
& c 3=c 3 \text { ant } 1 .
\end{aligned}
$$

The improved scheme can be easily extended to multiple antennas.

\section{B. External-calibration}

External calibration refers to the terminal selecting BS as the supporter [15]. During the calibration phase, they transmit the dedicated test signals to each other. Using channel estimation, the terminal get the downlink CSI $h_{d}$ and BS get the uplink CSI $h_{u}$ respectively. And then BS feedback $h_{u}$ to 
the terminal and the terminal compute the calibration factor using $k=h_{d} / h_{u}$.

\section{Cooperative Calibration}

Cooperative calibration refers to the terminal select other terminal as the supporter. Under the control of the base station, the terminal compensates the RF mismatches through another terminal. When the terminal is at cell edge, using externalcalibration, calibration accuracy deteriorates in case of low SNR due to channel measurement error [14]. Based on cooperative communication ideas, the BS measures average SNR $\Gamma_{B S}$ of the terminal request signal firstly. If $\Gamma_{B S}$ is less than a threshold level $\Gamma_{t h}$, the BS find out a feasible supporter which have higher $\operatorname{SNR} \Gamma_{S}$ than $\Gamma_{B S}$ around the terminal. Afterward, the terminal compensates the RF mismatches through the feasible supporter using relative calibration.

The above three schemes are based on relative calibration. The differences of them are the choice of supporter. In the selfcalibration, the overhead is low and no additional channel feedback is needed. However, in order to avoid the low noise amplifier (LNS) saturated, the transmit power of the calibration signal is smaller than that of data transmission, so accuracy of self-calibration is affected by power-dependent analog devices. External calibration doesn't have such a problem, because calibration pilot signal in external calibration has the same transmit power as in data transmission. The drawback is that a distant terminal from the BS suffers from insufficient calibration accuracy. Though cooperative calibration can solve this problem, further researches on it are needed due to the fact that there are complexity and safety issues to be resolved in collaborative communication. In addition, the above three schemes can use in mixed mode, like literature [14] which proposes a new control scheme to adaptively select external calibration mode or cooperative calibration mode depending on the terminal's situation.

\section{CONCLUSION}

In order to exploit the channel reciprocity between uplink and downlink in TDD system, calibrations at UE and at BS are needed. CE can be expressed as a diagonal matrix whose elements meet the different statistics distributions. As far as the $\mathrm{CE}$ at UE is concerned, only amplitude error will impact the system performance. Relative calibration based on digital signal processing has been extensively studied due to its low cost and flexibility. As far as the calibration in terminal is concerned, there are three possible methods including selfcalibration, external-calibration and cooperative calibration. How to carry out calibration to get the tradeoff between system performance and complexity needs further study.

\section{REFERENCES}

[1] N. Promsuvana and P. Uthansakul, "Feasibility of adaptive $4 \times 4$ MIMO system using channel reciprocity in FDD mode," in Communications, 2008. APCC 2008. 14th Asia-Pacific Conference on, 2008, pp. 1-5.

[2] M. Guillaud, D. Slock, and R. Knopp, "A practical method for wireless channel reciprocity exploitation through relative calibration," Proc. of ISSPA 2005, Sept. 2005.

[3] G.Jiann-Ching and L. D. Larsson, "Modeling and evaluation of MIMO systems exploiting channel reciprocity in TDD mode," in Vehicular Technology Conference, 2004. VTC2004-Fall. 2004 IEEE 60th, 2004, pp. 4265-4269 Vol. 6.

[4] 3GPP ,R1-084287,CATT, "UL SU-MIMO Antenna Calibration at UE", Nov,2008.

[5] A.Bourdoux, B. Come, and N. Khaled, "Non-reciprocal transceivers in OFDM/SDMA systems: impact and mitigation," in Radio and Wireless Conference, 2003. RAWCON '03. Proceedings, 2003, pp. 183-186.

[6] V.Jungnickel, V. Kr"uger, G. Istoc, T. Haustein, and C. von Helmolt, "A MIMO system with reciprocal transceivers for the time-division duplex mode," in Proc. IEEE Antennas and Propagation Society Symposium, June 2004, vol. 2, pp.1267-1270.

[7] 3GPP,R1-091753, "LTE DL Beamforming performance and evolution to dual stream", Nokia, Nokia Siemens Networks.

[8] 3GPP,R1-091754," Discussion on dual layer beamforming", Nokia, Nokia Siemens Networks.

[9] 3GPP, R1-091794, "Hardware calibration requirement for dual layer beamforming", Huawei

[10] Y.Hara, Y. Yano, and H. Kubo, "Antenna array calibration using frequency selection in OFDMA/TDD systems," Proc. of Globecom'08,Dec. 2008.

[11] J.Liu, A. Bourdoux, J. Craninckx, P. Wambacq, B. Come, S. Donnay, and A. Barel, "OFDM-MIMO WLAN AP front-end gain and phase mismatch calibration," in Radio and Wireless Conference, 2004 IEEE, 2004, pp. 151-154.

[12] L. Jian, G. Vandersteen, J. Craninckx, M. Libois, M. Wouters, F. Petre, and A. Barel, "A novel and low-cost analog front-end mismatch calibration scheme for MIMO-OFDM WLANs," in Radio and Wireless Symposium, 2006 IEEE, 2006, pp. 219-222.

[13] K.Nishimori,K.Cho,Y.Takatori,and T. Hori , "Automatic Calibration Method Using Transmitting Signals of an Adaptive Array for TDD Systems".

[14] Y. Hara, Y. Yano, and H. Kubo, "Cooperative Control for Antenna Array Calibration in OFDMA/TDD Systems" Proc. of Globecom'08,Dec. 2009.

[15] US20090186590 method for channel calibration.

[16] US20100067588 wireless communication apparatus,antenna calibration method and computer program. 